\title{
Esterification Product Protection Strategies for Direct and Selective Methane Conversion
}

\author{
Andrea N. Blankenship§a, Manoj Ravi ${ }^{\mathrm{a}}$ and Jeroen A. van Bokhoven ${ }^{\star a, b}$ \\ §SCS-DSM award for best virtual poster in Catalysis Sciences \& Engineering (runner-up)
}

\begin{abstract}
A scale-flexible process for the direct and selective oxidation of methane to primary oxygenates is of great interest, however, a commercially feasible approach has yet to be realized due to a number of challenges. Low product yields imposed by a well-established selectivity-conversion limit are particularly burdensome for direct methane-to-methanol chemistry. One strategy that has emerged to break out of this limit is the in situ esterification of produced methanol to the more oxidation-resistant methyl ester. However, these methaneto-methyl-ester approaches still elude commercialization despite their unprecedented high yields. Herein, we outline some of the key barriers that hinder the commercial prospects of this otherwise promising route for highyield direct catalytic methane conversion, including extremely corrosive reagents, homogeneous catalysts, and inviable oxidants. We then highlight directions to address these challenges while maintaining the characteristic high performance of these systems. These discussions support the efficacy of product protection strategies for the direct, selective oxidation of methane and encourage future work in developing creative solutions to merge this promising chemistry with more practical industrial requirements.
\end{abstract}

Keywords: Catalysis · Methanol · Methyl ester · Partial oxidation of methane $\cdot$ Product protection

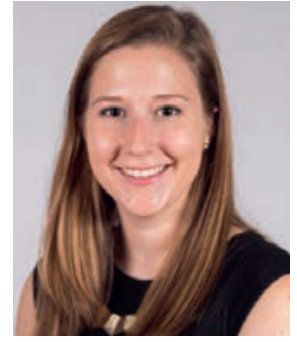

Andrea Blankenship earned her Bachelor's degree in Chemical Engineering at the Massachusetts Institute of Technology in 2018. Thereafter, she completed a Master's degree in Chemical and Bioengineering at ETH Zurich in 2020. She is currently a PhD student in the group of Prof. Jeroen A. van Bokhoven, focusing on employing product protection strategies for the direct catalytic partial oxidation of methane.

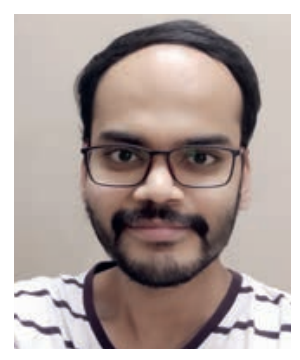

Manoj Ravi obtained his BTech degree in Chemical Engineering from AC Tech, Anna University, India in 2014 and an MSc in Chemical and Bioengineering from ETH Zurich in 2016. He was the recipient of the Willi Studer Prize for the best student of the Master degree program. Subsequently, he stayed on at ETH Zurich to pursue his $\mathrm{PhD}$ under the supervision of Prof. Jeroen A. van Bokhoven, which he successfully completed in 2020.

\section{Direct Catalytic Methane Valorization - Challenges and Opportunities}

Methane, the primary component of natural gas, is an important and versatile feedstock for commodity chemicals and liquid energy carriers, including methanol, ammonia, and olefins. ${ }^{[1]}$ On large industrial scales, the valorization of methane occurs typically through 'indirect' pathways that first convert methane to syngas, and this syngas is subsequently used in additional steps to produce the desired product. However, the large capital- and energy-intensities associated with this syngas production are costprohibitive for smaller scale operations $(<2.5 \mathrm{kt} / \mathrm{d}))_{.[1 \mathrm{a}, \mathrm{e}, 2]}$ The drive to address this gap in methane valorization technology has been further fueled in recent decades by the concomitant discovery and development of unconventional shale reserves for oil and natural gas. ${ }^{[1 c]}$ The opportunities for more sustainably produced fuels and high-value chemicals from these substantial methane sources are currently underexploited due to the lack of practical conversion and transportation technologies for these small, remote, and decentralized sites, constituting a technology gap that ultimately leads to large volumes of flared 'waste' methane. ${ }^{[2 a, 3]}$ 'Direct' methane conversion processes, referring to non-syngasbased routes such as the partial oxidation to primary oxygenates, have been heavily invested in by both the academic and industrial communities as a scale-flexible solution. ${ }^{[4]}$ These efforts have revealed an array of chemistry and engineering challenges that have precluded the emergence of a viable commercial solution, a reason why direct, selective methane conversion is often referred to as a 'Holy Grail' of catalysis. ${ }^{[4 a, 5]}$

Out of a great diversity of potential approaches for such a challenge, one frequently explored route is the partial oxidation of methane directly to methanol or methanol derivatives using homogeneous, heterogeneous, or biological enzyme catalytic systems. ${ }^{[1 \mathrm{~d}, 4 \mathrm{a}]}$ An overview of their performance based on prod-

\footnotetext{
${ }^{*}$ Correspondence: Prof. J. A. van Bokhoven ${ }^{\text {ab }}$, E-mail: jeroen.vanbokhoven@chem.ethz.ch 
uct yield is shown in Fig. 1. Notably, systems that catalyze the conversion of methane to methanol, whether homogeneously or heterogeneously, display low product yields that are essentially independent of the specific catalyst employed. This is largely in part due to the issue that direct methane to methanol conversion hinges upon the recovery of a product that is severely susceptible to over-oxidation, establishing a selectivity-conversion paradigm that does not allow for practical yields and conditions for a realistic commercial process. ${ }^{[4 a, 6]}$ This limit underscores the necessity of some form of 'product protection' in order to achieve high product yields. Product protection can be accomplished through physical or chemical means, whereby methanol is sequestered from oxidizing reagents/sites or chemically functionalized to a more stable derivative, respectively.

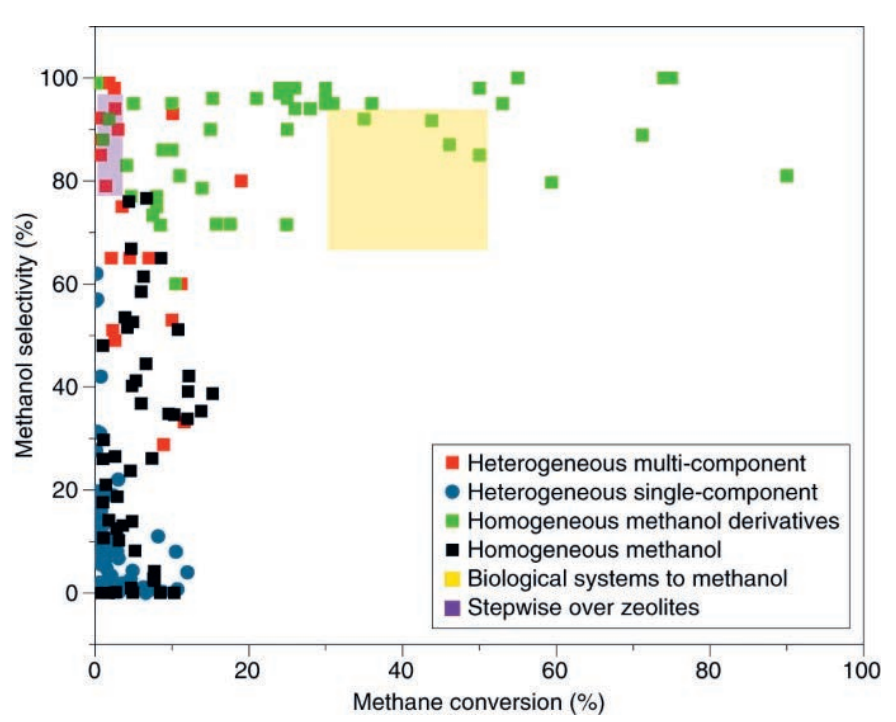

Fig. 1. Summarizing the selectivity-conversion performance of different methane oxidation systems. Figure reproduced from ref. [25] with permission of the Publisher.

One compelling strategy is the chemical protection of methanol within an ester that is less prone to further oxidation. Initial studies demonstrated the selectively catalyzed partial oxidation of methane with homogeneous transition-metal catalysts in concentrated sulfuric acid to produce methyl bisulfate (see Eqn. (1) ) with favorable yields (surpassing 70\%) and high turnover frequencies using this approach. ${ }^{[7]}$ Kinetic studies suggest that the oxidation rate for methyl bisulfate can be up to 100 times less than that of methane under the reaction conditions. ${ }^{[7 b]}$

$$
\mathrm{CH}_{4}+2 \mathrm{H}_{2} \mathrm{SO}_{4} \stackrel{\text { cat. }}{\rightarrow} \mathrm{CH}_{3} \mathrm{OSO}_{3} \mathrm{H}+2 \mathrm{H}_{2} \mathrm{O}+\mathrm{SO}_{2}
$$

$$
\mathrm{CH}_{3} \mathrm{OSO}_{3} \mathrm{H}+\mathrm{H}_{2} \mathrm{O} \rightarrow \mathrm{CH}_{3} \mathrm{OH}+\mathrm{H}_{2} \mathrm{SO}_{4}
$$

In addition, catalytic systems employing this same approach with trifluoroacetic acid to esterify methanol to methyl trifluoroacetate with high selectivities and yields were published. ${ }^{[8]}$ Different oxidants can be used for this system (see Table 1), and the reaction stoichiometry with molecular oxygen as the oxidant is shown in Eqn. (3):

$$
\mathrm{CH}_{4}+\frac{1}{2} \mathrm{O}_{2}+\mathrm{CF}_{3} \mathrm{COOH} \stackrel{\text { cat. }}{\rightarrow} \mathrm{CF}_{3} \mathrm{COOCH}_{3}+\mathrm{H}_{2} \mathrm{O}
$$

$$
\mathrm{CF}_{3} \mathrm{COOCH}_{3}+\mathrm{H}_{2} \mathrm{O} \rightarrow \mathrm{CH}_{3} \mathrm{OH}+\mathrm{CF}_{3} \mathrm{COOH}
$$

For both chemistries, the product ester can be hydrolyzed to obtain methanol as the final product (see Eqn. (2) and Eqn. (4)). These routes that produce a stable methyl derivative benefit from high selectivities independent of the methane conversion, even surpassing the methane-based yields of efficient bioenyzmatic systems that are regarded as the benchmarks of direct methane conversion (Fig. 1). Based on these findings, product protection via esterification emerges as a promising strategy to break out of the selectivity-conversion limit defining direct methane-to-methanol conversion in order to attain high product yields.

\section{Methanol Protection via Esterification - Key Challenges}

While the concept underlying product protection via methanol esterification has been proven successful at obtaining unprecedented product yields (Fig. 1), none of the reported processes employing this chemistry have been commercially viable to date. This highlights the fact that although high yields per pass are a key parameter for commercial prospect, it is important to think beyond this single criterion and assess the process more holistically, considering the relative cost of materials, compositions and sizes of separation and recycle streams, and required heat duties. ${ }^{[9]}$ Herein, we summarize a selection of common shortcomings of methane-to-methyl-ester (MTME) systems. It should be noted that rigorous process analyses are ultimately required to fully assess a proposed process scheme. The following points, however, serve as guidelines for maximizing the potential success of a process using this chemistry. The following list is not exhaustive of all potential issues in MTME processes, but rather an attempt to target a few relevant shared barriers. More detailed estimates of specific performance targets for methane conversion processes are described elsewhere.[9b,10]

\subsection{Corrosive and Concentrated Acid Solvents}

A crux of the MTME protection approach is the use of strong acid that is capable of esterifying methanol under the reaction conditions. It is not by chance that MTME conversion literature centers around the use of sulfuric acid/oleum and trifluoroacetic acid, as both acids are stable under the elevated temperatures and are effective at esterifying methanol to the corresponding stable ester in order to maintain high selectivities. The stability of the acid solvent is essential, as the substantial degradation of the acid medium would have negative consequences on material costs and potentially impact the reaction. This can be illustrated in the case of replacing trifluoroacetic acid with acetic acid, a substitution that leads to the ester formation primarily from the degradation of acetic acid rather than the conversion of methane. ${ }^{[8 \mathrm{~d}]}$ Acids such as trifluoroacetic acid and sulfuric acid are not as readily degraded and are therefore good candidates for the acid solvent. Furthermore, the strongly acidic and poorly coordinating nature of concentrated acids is essential for curbing the inhibition of the $\mathrm{CH}$ activation for some catalytically-active complexes by preventing the coordination of water and methanol to the complex.[9b] For the well-studied Catalytica Pt-complex in methyl bisulfate systems, a decrease in the sulfuric acid concentration from $100 \%$ to $99 \%$ results in an order of magnitude decrease in the obtainable yield at the same selectivity. ${ }^{[6 b]}$ Consequently, sufficiently high concentrations of the selected acid are required to both drive forward the esterification reaction and ensure the high activity of the homogeneous complexes.

Nonetheless, working with these strong acids in an extremely concentrated form can present a number of additional operational and material challenges. The highly corrosive nature of the reac- 
tion medium may pose greater health and environmental hazards and necessitates upgraded materials for the construction of vessels to reduce equipment wear, which can add significantly to the associated capital investment costs. ${ }^{[9]}$ Also, the sensitivity of some methyl bisulfate systems to even small amounts of water in the concentrated sulfuric acid or oleum medium raises additional concerns for the heat duties required to dry the acid, especially after the recovery of the product via hydrolysis that generally results in a large fraction of water in the recycled acid stream. ${ }^{[9]}$ Hydrolyzing the ester in the concentrated acid stream poses its own challenges, as the high exothermicity can cause the unintended evaporation of methanol and must be performed with caution to ensure safe handling. ${ }^{[11]}$ In short, although the strong acid mediums are beneficial for achieving high yields in this chemistry, there are a number of process-oriented consequences that deserve further consideration and motivate better management of the acid solvent.

\subsection{Homogeneous Catalysts}

Another general shortcoming of a majority of MTME systems is their reliance on homogeneous catalysts. Table 1 lists some prominent examples of homogeneous systems for this chemistry, which greatly outnumber the very limited published solid catalyzed systems discussed further in Section 3.2. For systems that convert methane to methyl bisulfate in a liquid medium of oleum or concentrated sulfuric acid, molecular noble metal catalysts have been the most extensively explored and optimized (Table 1, Entries 1-4). To prevent the decomposition of the platinum and palladium catalysts to reduced bulk states or insoluble salts under the aggressive reaction conditions, various ligands are used to form active and stable metal complexes. ${ }^{[7 b]}$ Simpler Pt- and Pd salts have also subsequently been successfully demonstrated for this chemistry, maintaining high stability and selectivity at high turnovers. ${ }^{[7 c, 10 a]}$ In the methyl trifluoroacetate systems, a variety of diverse homogeneous catalysts have been proposed, ranging from noble metal complexes to heteropolyacids to simpler transition metal salts (Table 1).

Although gas-liquid processes with a molecular catalyst are conceivable for alkane to alcohol conversion, ${ }^{[12]}$ they generally pose greater catalyst recovery and regeneration difficulties than those catalyzing the conversion heterogeneously. The homogeneity of the catalyst necessitates additional downstream processing steps for the separation and regeneration of the catalyst, which is generally not encouraged given the economics of lower alkane conversion. [9a] The fast and irreversible deactivation of catalysts encountered in some systems is also concerning. In methyl trifluoroacetate systems, the formation of inactive metal fluorides from transition-metal salts can lead to the deactivation of the catalyst in under 2-3 hours of reaction. ${ }^{[8 c, d]}$ Some catalysts may also require regeneration of the higher-valent transition-metal species through an acid-base swing process, which is acknowledged to be impractical for a viable MTME process. ${ }^{[8 c, e]}$ Each of these issues highlights notable shortcomings in homogeneous catalysts for MTME approaches and the necessity to explore solid catalysts with improved stability, performance, and recoverability.

\subsection{Uneconomical Oxidants}

A feature of many current MTME systems that hinders their commercial applicability is their reliance on relatively expensive oxidants. High product yields are often coupled with the use of strong oxidants, including hydrogen peroxide or potassium per-

Table 1. Overview of a selection of published homogeneous methane-to-methyl-ester systems.

\begin{tabular}{|c|c|c|c|c|c|c|c|}
\hline [Pre]catalyst & Oxidant & $\mathbf{P}_{\text {methane }}[$ bar $]$ & Temp. $\left[{ }^{\circ} \mathbf{C}\right]$ & Max. Yield [\%] & TO a (Time [h]) & Max. Ester Conc. [M] & Ref. \\
\hline \multicolumn{8}{|c|}{ Methyl Bisulfate } \\
\hline$[\mathrm{bpym}] \mathrm{PtCl}_{2}$ & $\mathrm{H}_{2} \mathrm{SO}_{4} / \mathrm{SO}_{3}$ & 34 & 220 & 72 & $>500(2.5 \mathrm{~h})$ & $\sim 1$ & {$[7 b]$} \\
\hline $\mathrm{Pd}$ & $\mathrm{SO}_{3}$ & 45 & 160 & 25 & 17.7 (18 h) & 0.5 & {$[7 \mathrm{c}]$} \\
\hline $\mathrm{I}_{2}$ & $\mathrm{SO}_{3}$ & 40 & 180 & 70 & $>200(2.5)$ & $>2$ & {$[7 d]$} \\
\hline $\mathrm{K}_{2} \mathrm{PtCl}_{4}$ & $\mathrm{SO}_{3}$ & $>53$ & 215 & $<30$ & $>16,000(2 \mathrm{~h})$ & $\sim 1$ & [10a] \\
\hline \multicolumn{8}{|c|}{ Methyl Trifluoroacetate } \\
\hline $\mathrm{H}_{5} \mathrm{PV}_{2} \mathrm{Mo}_{10} \mathrm{O}_{40}$ & $\mathrm{~K}_{2} \mathrm{~S}_{2} \mathrm{O}_{8}$ & $5-20$ & 80 & 78 & $<142(20 \mathrm{~h})$ & 0.3 & [13a] \\
\hline $\mathrm{Cu}(\mathrm{OAc})_{2}$ & $\mathrm{~K}_{2} \mathrm{~S}_{2} \mathrm{O}_{8}$ & 5 & 100 & 91 & $<151(20 \mathrm{~h})$ & 0.8 & [13b] \\
\hline $\mathrm{H}_{4} \mathrm{PV}_{1} \mathrm{Mo}_{11} \mathrm{O}_{40}$ & $\mathrm{H}_{2} \mathrm{O}_{2}$ & 50 & 80 & $<4.4^{\mathrm{b}}$ & $224^{\mathrm{b}}(24 \mathrm{~h})$ & $0.05^{b}$ & [26] \\
\hline Pd-NHC complex & $\mathrm{K}_{2} \mathrm{~S}_{2} \mathrm{O}_{8}$ & $20-30$ & 80 & $<5$ & $30(14 \mathrm{~h})$ & $<0.1$ & {$[8 \mathrm{~b}]$} \\
\hline$\left[\mathrm{Pd}(\text { hfacac })_{2}\right]$ & $\mathrm{H}_{2} \mathrm{O}_{2}$ & 30 & $50-75$ & 13.7 & $50.1(4 \mathrm{~h})$ & $<0.2$ & [27] \\
\hline $\mathrm{Co}(\mathrm{OAc})_{2} \cdot 4 \mathrm{H}_{2} \mathrm{O}$ & $\mathrm{O}_{2}$ & 20 & 180 & 50 & $13.2(24 \mathrm{~h})$ & 0.5 & {$[8 \mathrm{~d}]$} \\
\hline $\mathrm{Mn}_{2} \mathrm{O}_{3}$ & $\mathrm{O}_{2}$ & 6.9 & 180 & 36 & $8.5(3 \mathrm{~h})$ & 0.01 & {$[8 \mathrm{c}]$} \\
\hline $\mathrm{CuO}$ & $\mathrm{K}_{2} \mathrm{~S}_{2} \mathrm{O}_{8}$ & 5.2 & 90 & 56 & $33(17 \mathrm{~h})$ & $0.12^{\mathrm{c}}$ & {$[8 \mathrm{e}]$} \\
\hline
\end{tabular}


sulfate (see Table 1), that are generally more expensive than the product ester or methanol. A basic comparison of feedstock costs reveals essentially no margin for any oxidant beyond pure $\mathrm{O}_{2}$, even before considering the other costs of the process. ${ }^{[9]}$ The cost analysis for methyl bisulfate systems that typically feature $\mathrm{SO}_{3}$ as the oxidant is nuanced by the proposal that $\mathrm{SO}_{3}$ could be regenerated from the formed $\mathrm{SO}_{2}$, thereby closing the cycle. Although the recycling of $\mathrm{SO}_{2} / \mathrm{SO}_{3}$ has been demonstrated on industrial scale, whether this oxidant recycling scheme is practical on a scale more relevant to the target applications of direct methane conversion processes is still unclear. This is further complicated by the fact that $\mathrm{SO}_{3}$ could react with trace water formed in the partial oxidation of methane to produce $\mathrm{H}_{2} \mathrm{SO}_{4}$, effectively resulting in losses of the oxidant during reaction.[9a] Given also the observed dependence of the catalytic productivity on the $\mathrm{SO}_{3}$ concentration in these systems, ${ }^{[10]}$ this side reaction should not be overlooked, especially at high methane conversions. Similar sensitivity of oxidants to trace water in methyl trifluoroacetate systems is generally not observed, and often trifluoroacetic anhydride is used as a convenient desiccant to avoid catalyst deactivation by water or hydrate formation. ${ }^{[8 b, 13]}$ Regardless of the specifics, it is evident that a greater emphasis should be placed on evaluating catalytic systems that are capable of conversion with a more economically reasonable final oxidant, primarily $\mathrm{O}_{2}$.

\section{Designing Improved Methane-to-Methyl-Ester Processes}

With a few of the major shortcomings of MTME systems outlined in detail, we now focus on methods to address these key challenges. The following discussions highlight interesting developments from the broader field of methane conversion and consider their efficacy thus far or applicability to MTME systems.

\subsection{Suitable Diluents for Acid Solvents}

As stated previously, the reagents involved in MTME processes are largely disfavored for commercial lower alkane conversion both from the standpoint of the expensive oxidants and the highly corrosive and aggressive liquid acid solvents. Efforts focused on mitigating the challenges of the acid solvent are relatively sparse, as simply diluting the acid solvent is not a straight-forward task. Many common solvents miscible with the acids are likely to be unstable at elevated temperatures under protic and oxidizing conditions, especially if they contain functional groups that could be reactive in these environments. As an example, a study on catalytic alkane oxidation with hydrogen peroxide in liquid acetonitrile and trifluoroacetic acid reported substantial oxidation of the acetonitrile despite the relatively mild conditions $\left(<80^{\circ} \mathrm{C}\right) .{ }^{[14]}$ These criteria severely restrict the selection of suitable solvents, although a few options have appeared over the years. An interesting class of solvents that have been successfully demonstrated for MTME chemistry is ionic liquids. Tang and coworkers were the first to use ionic liquids in combination with the chemistry and homogeneous catalysis of the Catalytica system. Various ionic liquids were mixed with sulfuric acid and Pt salts to create a ternary system that was thermally stable, dissolved Pt salts that were insoluble in the conventional system, promoted the reactivity of $\mathrm{Pt}$, and improved the water tolerance of the system overall. ${ }^{[15]}$ In following years, Lin and coworkers incorporated an ionic liquid into the liquid-phase oxidation of methane to methyl trifluoroacetate using potassium persulfate and a solid Au-based catalyst, in which they attributed improved performance to the promoting and stabilizing effects of the ionic liquid on the catalyst. ${ }^{[16]}$ The large variety of ionic liquids with different physical properties is appealing, as it allows for greater flexibility in selecting an appropriate and stable compound for different MTME systems. These results also underscore the benefits a co-solvent can provide beyond acting purely as a diluent in the system, given that the co- solvent can be efficiently recycled in order to minimize the impact of the additional material costs.

In addition to ionic liquids, other compounds have been used in methane conversion to dilute the acid solvent while enhancing performance, for example, by increasing the uptake of methane or other reacting gases into the liquid phase. This particular effect was shown by Yokoi and coworkers for the liquid-phase conversion of methane to methanol using hydrogen peroxide dissolved in a sulfolane/water solvent, a mixture that was both stable and enabled higher concentrations of dissolved methane in the liquid medium. ${ }^{[17]}$ It should be noted that for MTME systems, water is generally unsuitable as a diluent because a large concentration would be expected to drive the hydrolysis of the methyl ester, thereby deprotecting methanol and allowing greater rates of overoxidation. Specifically for methyl bisulfate systems, the introduction of excess water is especially problematic for the function of the catalyst, in which even concentrations of sulfuric acid just below $90 \%$ can severely impede productivity. ${ }^{[9 b]}$ Nonetheless, the sulfolane/water mixture illustrates the potential to use an additional co-solvent to enhance the reaction environment in order to improve productivity.

On the other side, systems using trifluoroacetic acid may benefit from the exploitation of the interesting properties of fluorine chemistry. Although trifluoroacetic acid is not entirely 'fluorous' in nature, it is miscible in fluorous solvents such as perfluoroalkanes, materials that are inert and extremely stable even under aggressive oxidizing conditions. ${ }^{[18]}$ Wang and co-workers observed 5-fold improvements in product yield for homogeneously-catalyzed direct methane partial oxidation upon adding a relatively small quantity of perfluorooctane $(<20 \mathrm{vol} . \%)$ to the trifluoroacetic acid solvent. ${ }^{[19]}$ The improved yields were attributed to the improved mass transfer afforded by the enhanced solubility of gases in the perfluorooctane. This again emphasizes the benefits of using a co-solvent in MTME systems to not only create milder conditions, but also promote better performance. Overall, both MTME chemistries have at least a few options of co-solvents or diluents that stand not only to mitigate the issues created by the use of harsh undiluted acids, but also to improve the systems in terms of catalyst stability and productivity.

\subsection{Solid Catalysts}

The transition to solid catalysts is a primary goal in this chemistry and has already seen some promising recent success, despite the limited number of publications to date. Heterogeneous catalysts are attractive for this chemistry because they may greatly simplify the separation and catalyst regeneration schemes in a continuously operating process, which would have a positive effect on the overall process costs. The design of new catalysts must consider structure-function relationships that determine and relate high selectivity, high stability, and high productivity without compromise on any of these fronts. ${ }^{[9 b]}$ One of the first and best demonstrations of heterogeneously-catalyzed methaneto-methyl-ester conversion by Schüth and coworkers translated the platinum coordination of the molecular Catalytica catalyst to platinum coordination on a solid carbon support for the conversion of methane to methyl bisulfate in oleum and achieved impressive turnovers. ${ }^{[20]}$ At the time of writing, a new, highly active porous organic polymer-supported Pd catalyst for the conversion of methane to methyl trifluoroacetate at lower temperatures $\left(80{ }^{\circ} \mathrm{C}\right)$ with potassium persulfate has been proposed by Yuan and $\mathrm{Su}$ et al. ${ }^{[21]}$ In both cases, the strong catalytic performance comes as a result of replicating active motifs from homogeneous analogues while reaping the additional benefits of a stable solid support with tuneable properties (e.g. porosity, hydrophobicity). Although the development of active solid catalysts for methane-to-methyl-ester chemistries has historically remained a challenge, in large part due to the harsh reaction conditions, the recent progress underscores 
the possibility in overcoming these challenges with thoughtful material design. In congruence with improvements in reagents and reaction conditions, the development of solid catalysts deserves significant attention as a means to improving the commercial applicability of these processes.

\subsection{Viable Oxidants}

Lastly, the feedstock cost is a clearly bounded parameter that restricts the choice of the oxidant to $\mathrm{O}_{2}$ or water. Instances of aerobic methane conversion in homogeneous systems are demonstrated, but these systems generally lag in turnovers compared to others that employ strong oxidants (see Table 1), motivating the further development of systems that can operate using $\mathrm{O}_{2}$ without compromising good performance. Alternative approaches to this goal have been proposed that use $\mathrm{O}_{2}$ indirectly as the oxidant for methane partial oxidation. Hydrogen peroxide or derivatives have been synthesized in situ through co-feeding $\mathrm{H}_{2} / \mathrm{O}_{2}$ mixtures. ${ }^{[22]}$ An early demonstration of this approach by Mizuno et al. used a feed composition $28 \% \mathrm{CH}_{4} /$ $33 \% \mathrm{H}_{2} / 14 \% \mathrm{O}_{2} / 14 \% \mathrm{~N}_{2}$ for the catalytic conversion of methane to formic acid at low temperatures with a palladium heteropolycomplex. ${ }^{[22 a]}$ Critically, the obtained methane conversions were well below $1 \%$ for this system. In addition, Yang and Xiao et al. recently revealed a hybrid material that was able to localize in situ $\mathrm{H}_{2} \mathrm{O}_{2}$ generation from an $\mathrm{O}_{2} / \mathrm{H}_{2}$ mixture $\left(\mathrm{H}_{2} / \mathrm{CH}_{4}\right.$ ratio $\left.\sim 2\right)$ and subsequently catalyze the selective oxidation of methane.[23] This strategy aims to alleviate challenges associated with the relatively quick degradation of $\mathrm{H}_{2} \mathrm{O}_{2}$, while creating high local concentrations of $\mathrm{H}_{2} \mathrm{O}_{2}$ near oxidation sites. ${ }^{23]}$ However, given that the price of sustainably-produced hydrogen gas that would either need to be supplied or produced on-site (e.g. via electrolysis) is in most instances more expensive than methane, these strategies fail to address the root of the oxidant challenge in their current form, especially when above stoichiometric $\mathrm{H}_{2} / \mathrm{CH}_{4}$ feed ratios are used to attain appreciable methane conversion. At best, the considerable cost of the feedstock gas mixture necessitates extremely efficient conversion of $\mathrm{O}_{2} / \mathrm{H}_{2}$ to $\mathrm{H}_{2} \mathrm{O}_{2}$ coupled with high conversions of the oxidant to the product as a minimum requirement for systems using this oxidant scheme. Although the cost-effectiveness of this in situ generation needs to be further considered, it nevertheless represents a creative approach to the issue of oxidants in these systems. A second strategy is the use of an air-recyclable oxidant as an oxygen transfer agent between $\mathrm{O}_{2}$ and methane. This scheme is commonly proposed for the recycling of $\mathrm{SO}_{3}$ in methyl bisulfate systems as mentioned previously, and more recently a catalytic system for the conversion of methane to $\mathrm{DME}$ with $\mathrm{NO}_{2}$ produced in the couple gas phase reaction of $\mathrm{NO}$ and $\mathrm{O}_{2} \cdot{ }^{[24]} \mathrm{In}$ both cases, it is crucial that the recycling scheme does not pose complicated gas separation with burdensome energy duties or substantial purges of the valuable feedstocks. Overall, these approaches that indirectly use $\mathrm{O}_{2}$ as the final oxidant are conceptually interesting, yet their practicality relies on the ultimate ability to address these process-oriented issues.

\section{Conclusion}

Progress over the previous decades in direct methane conversion technologies, while still far from meeting industrial targets, has resulted in the development of chemical strategies that allow high-yield methane functionalization with high selectivity. Product protection via the esterification of methanol to a more stable product is a well-demonstrated strategy to obtain high yields, but this often comes at the cost of harsh undiluted acid solvents, reliance on homogeneous catalysts, and cost-prohibitive oxidants, all factors that contribute to a number of challenges for commercialization. Only by developing a solution that addresses these shortcomings can the goal of a highly productive heterogeneously-catalyzed process using economical reagents and milder reaction conditions be reached.

\section{Acknowledgements}

This work was supported financially by ETH Zurich and the Swiss National Science Foundation (Grant No. 200021_178943). The authors thank the SCS and DSM Nutritional Products for their generous sponsorship of the award.

Received: January 31, 2021

\begin{tabular}{llrrrrr}
\hline$[1]$ & a) J. P. Lange, Catal. & Today & 2001, & 64, & 3, \\
& https://doi.org/10.1016/S0920-5861(00)00503-4; & b) & C.
\end{tabular} https://doi.org/10.1016/S0920-5861(00)00503-4;
Hammond, S. Conrad, I. Hermans, ChemSusChem 2012, 5, 1668 , https://doi.org/10.1002/cssc.201200299; c) E. McFarland, Science 2012, 338, 340, https://doi.org/10.1126/science.1226840; d) A. I. Olivos-Suarez, A. Szecsenyi, E. J. M. Hensen, J. Ruiz-Martinez, E. A. Pidko, J. Gascon, ACS Catal. 2016, 6, 2965, https://doi.org/10.1021/acscatal.6b00428; e) J. R. Rostrup-Nielsen, J. Sehested, J. K. Norskov, Adv. Catal. 2002, 47, 65, https://doi.org/10.1016/S0360-0564(02)47006-X.

[2] a) J. P. Lange, Ind. Eng. Chem. Res. 1997, 36, 4282 https://doi.org/10.1021/ie9607762; b) I. Dybkjaer, K. Aasberg-Petersen, Can. J. Chem. Eng. 2016, 94, 607, https://doi.org/10.1002/cjce.22453.

[3] a) S. Mokhatab, J. Mak, J. V. Valappil, D. A. Wood, 'Handbook of liquefied natural gas', First edition. ed., Elsevier, GPP, Amsterdam, 2014; b) W. Taifan, J. Baltrusaitis, Appl. Catal. B: Environ. 2016, 198, 525, https://doi.org/10.1016/j.apcatb.2016.05.081; c) IEA, 'Tracking Fuel Supply', Paris, 2019.

[4] a) M. Ravi, M. Ranocchiari, J. A. van Bokhoven, Angew. Chem. Int. Ed. 2017, 56, 16464, https://doi.org/10.1002/anie.201702550; b) P. Tang, Q. J. Zhu, Z. X. Wu, D. Ma, Energ. Environ. Sci. 2014, 7, 2580, https://doi.org/10.1039/c4ee00604f; c) J. P. Lange, P. J. A. Tijm, Chem. Eng. Sci. 1996, 51, 2379, https://doi.org/10.1016/0009-2509(96)00094-2.

[5] B. A. Arndtsen, R. G. Bergman, T. A. Mobley, T. H. Peterson, Acc. Chem. Res. 1995, 28, 154, https://doi.org/10.1021/ar00051a009.

[6] a) J. P. Lange, K. P. de Jong, J. Ansorge, P. J. A. Tijm, in 'Studies in Surface Science and Catalysis', Vol. 107, Eds. M. de Pontes, R. L. Espinoza, C. P. Nicolaides, J. H. Scholtz, M. S. Scurrell, Elsevier, 1997, p. 81, https://doi.org/10.1016/S0167-2991(97)80320-5; b) M. Ahlquist, R. J. Nielsen, R. A. Periana, W. A. Goddard, J. Am. Chem. Soc. 2009 131, 17110, https://doi.org/10.1021/ja903930e; c) A. A. Latimer, A. Kakekhani, A. R. Kulkarni, J. K. Norskov, ACS Catal. 2018, 8, 6894, https://doi.org/10.1021/acscatal.8b00220.

[7] a) R. A. Periana, D. J. Taube, E. R. Evitt, D. G. Loffler, P. R. Wentrcek, G. Voss, T. Masuda, Science 1993, 259, 340, https://doi.org/10.1126/science.259.5093.340; b) R. A. Periana, D. J. Taube, S. Gamble, H. Taube, T. Satoh, H. Fujii, Science 1998, 280, 560, https://doi.org/10.1126/science.280.5363.560;

B. Michalkiewicz, Kinet. Catal+ 2003, 44, 801, https://doi.org/10.1023/B:KICA.0000009057.79026.0b; d) X. Gang, Y. M. Zhu, H. Birch, H. A. Hjuler, N. J. Bjerrum, Appl. Catal. A: Gen. 2004, 261, 91, https://doi.org/10.1016/j.apcata.2003.10.039.

[8] a) L. C. Kao, A. C. Hutson, A. Sen, J. Am. Chem. Soc. 1991, 113, 700, https://doi.org/10.1021/ja00002a063; b) M. Muehlhofer, T. Strassner, W. A. Herrmann, Angew. Chem. Int. Ed. 2002, 41, 1745, https://doi.org/10.1002/1521-3773(20020517)41:10<1745::AIDANIE1745>3.0.CO;2-E; c) W. S. Chen, J. A. Kocal, T. A. Brandvold, M. L. Bricker, S. R. Bare, R. W. Broach, N. Greenlay, K. Popp, J. T. Walenga, S. S. Yang, J. J. Low, Catal. Today 2009, 140, 157, https://doi.org/10.1016/j.cattod.2008.10.011; d) T. Strassner, S. Ahrens, M. Muehlhofer, D. Munz, A. Zeller, Eur. J. Inorg. Chem. 2013, 3659, https://doi.org/10.1002/ejic.201300213; e) M. Ravi, J. A. van Bokhoven, ChemCatChem 2018, 10, 2383, https://doi.org/10.1002/cctc.201800412.

[9] a) J.-P. Lange, in 'Sustainable Strategies for the Upgrading of Natural Gas: Fundamentals, Challenges, and Opportunities', Eds. E. G. Derouane, V. Parmon, F. Lemos, F. Ramôa Ribeiro, Springer Netherlands, 2005, p. 51; b) R. A. Periana, G. Bhalla, W. J. Tenn, K. J. H. Young, X. Y. Liu, O. Mironov, C. J. Jones, V. R. Ziatdinov, J. Mol. Catal. A: Chem. 2004, 220, 7 , https://doi.org/10.1016/j.molcata.2004.05.036.

[10] a) T. Zimmermann, M. Soorholtz, M. Bilke, F. Schuth, J. Am. Chem. Soc. 2016, 138, 12395, https://doi.org/10.1021/jacs.6b05167; b) J. P. Lange, V. L. Sushkevichk, A. J. Knorpp, J. A. van Bokhoven, Ind. Eng. Chem. Res. 2019, 58, 8674, https://doi.org/10.1021/acs.iecr.9b01407.

[11] B. Michalkiewicz, K. Kalucki, J. G. Sosnicki, J. Catal. 2003, 215, 14, https://doi.org/10.1016/S0021-9517(02)00088-X

[12] B. L. Conley, W. J. Tenn, K. J. H. Young, S. K. Ganesh, S. K Meier, V. R. Ziatdinov, O. Mironov, J. Oxgaard, J. Gonzales, W. A. Goddard, R. A. Periana, J. Mol. Catal. A: Chem. 2006, 251, 8, https://doi.org/10.1016/j.molcata.2006.02.035. 
[13] a) D.-g. Piao, K. Inoue, H. Shibasaki, Y. Taniguchi, T. Kitamura, Y Fujiwara, J. Organomet. Chem. 1999, 574, 116; b) G. C. Yin, D. G. Piao, T. Kitamura, Y. Fujiwara, Appl. Organomet. Chem. 2000, 14, 438, https://doi.org/10.1002/1099-0739(200008).

[14] D. Mandelli, A. C. N. do Amaral, Y. N. Kozlov, L. S. Shul'pina, A. J. Bonon, W. A. Carvalho, G. B. Shul'pin, Catal. Lett. 2009, 132, 235, https://doi.org/10.1007/s10562-009-0103-Z.

[15] J. H. Cheng, Z. W. Li, M. Haught, Y. C. Tang, Chem. Commun. 2006, 4617, https://doi.org/10.1039/b610328f.

[16] T. Li, S. J. Wang, C. S. Yu, Y. C. Ma, K. L. Li, L. W. Lin, Appl. Catal. A: Gen. 2011, 398, 150, https://doi.org/10.1016/j.apcata.2011.03.028.

[17] P. P. Xiao, Y. Wang, T. Nishitoba, J. N. Kondo, T. Yokoi, Chem. Commun. 2019, 55, 2896, https://doi.org/10.1039/c8cc10026h.

[18] W. A. Arnold, T. G. Hartman, J. McQuillen, J. Spacecraft Rockets 2007, 44, 94, https://doi.org/10.2514/1.22537.

[19] J. L. Yuan, L. L. Wang, Y. Wang, Ind. Eng. Chem. Res. 2011, 50, 6513, https://doi.org/10.1021/ie1018113.

[20] R. Palkovits, M. Antonietti, P. Kuhn, A. Thomas, F. Schuth, Angew. Chem. Int. Ed. 2009, 48, 6909, https://doi.org/10.1002/anie.200902009.

[21] Y. Zhang, M. Zhang, Z. Han, S. Huang, D. Yuan, W. Su, ACS Catal. 2021, 1008, https://doi.org/ 10.1021/acscatal.0c05205.

[22] a) J.-S. Min, H. Ishige, M. Misono, N. Mizuno, J. Catal. 2001, 198, 116, https://doi.org/10.1006/jcat.2000.3117; b) M. H. Ab Rahim, M. M. Forde, R. L. Jenkins, C. Hammond, Q. He, N. Dimitratos, J. A. Lopez-Sanchez, A. F. Carley, S. H. Taylor, D. J. Willock, D. M. Murphy, C. J. Kiely, G. J. Hutchings, Angew. Chem. Int. Ed. 2013, 52, 1280, https://doi.org/10.1002/anie.201207717; c) R. J. Lewis, A. Bara-Estaun, N.
Agarwal, S. J. Freakley, D. J. Morgan, G. J. Hutchings, Catal. Lett. 2019, 149, 3066, https://doi.org/10.1007/s10562-019-02876-7.

[23] Z. Jin, L. Wang, E. Zuidema, K. Mondal, M. Zhang, J. Zhang, C. Wang, X. Meng, H. Yang, C. Mesters, F.-S. Xiao, Science 2020, 367, 193, https://doi.org/10.1126/science.aaw1108.

[24] V. Vargheese, Y. Kobayashi, S. T. Oyama, Angew. Chem. Int. Ed. 2020, 59 , 16644, https://doi.org/10.1002/anie.202006020.

[25] M. Ravi, V. L. Sushkevich, A. J. Knorpp, M. A. Newton, D. Palagin, A B. Pinar, M. Ranocchiari, J. A. van Bokhoven, Nat. Catal. 2019, 2, 485 , https://doi.org/10.1038/s41929-019-0273-z.

[26] Y. Seki, J. S. Min, M. Misono, N. Mizuno, J. Phys. Chem. B 2000, 104 5940, https://doi.org/10.1021/jp000406y

[27] G. Ingrosso, N. Midollini, Mol. Catal. 2003, 204, 425, https://doi.org/10.1016/S1381-1169(03)00324-8

\section{License and Terms}

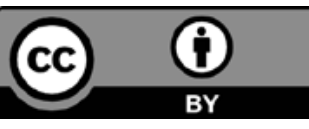

This is an Open Access article under the terms of the Creative Commons Attribution License CC BY 4.0. The material may not be used for commercial purposes.

The license is subject to the CHIMIA terms and conditions: (http:// chimia. ch/component/sppagebuilder/?view=page \&id=12).

The definitive version of this article is the electronic one that can be found at https://doi.org/10.2533/chimia.2021.305 